Research Paper

\title{
The Influence of Prednisone on the Efficacy of Cabazitaxel in Men with Metastatic Castration-Resistant Prostate Cancer
}

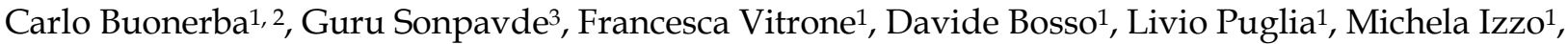 \\ Simona Iaccarino ${ }^{1}$, Luca Scafuri ${ }^{1}$, Margherita Muratore ${ }^{1}$, Francesca Foschini ${ }^{1}$, Brigitta Mucci ${ }^{1}$, Vincenzo

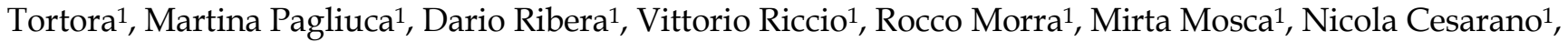 \\ Ileana Di Costanzo ${ }^{1}$, Sabino De Placido ${ }^{*}$, Giuseppe Di Lorenzo ${ }^{1 * \square}$
}

1. Department of Clinical Medicine and Surgery, University of Naples Federico II, Naples, Italy;

2. Istituto Zooprofilattico Sperimentale del Mezzogiorno, Portici, Italy;

3. University of Alabama at Birmingham Comprehensive Cancer Center, Birmingham.

* These two authors share senior authorship

$\triangle$ Corresponding author: Giuseppe Di Lorenzo, Phone: +39 081746 3660; Email: dilorengiuseppe@gmail.com Department of Clinical Medicine and Surgery, University of Naples Federico II, Naples, ItalyVia Pansini, 5 - Napoli (ITALY)

(C) Ivyspring International Publisher. This is an open access article distributed under the terms of the Creative Commons Attribution (CC BY-NC) license (https://creativecommons.org/licenses/by-nc/4.0/). See http://ivyspring.com/terms for full terms and conditions.

Received: 2017.03.12; Accepted: 2017.06.24; Published: 2017.08.22

\begin{abstract}
Background: Cabazitaxel is a second-generation taxane that is approved for use with concomitant low dose daily prednisone in metastatic castration resistant prostate cancer (mCRPC) after docetaxel failure. Since the role of daily corticosteroids in improving cabazitaxel efficacy or ameliorating its safety profile has not been adequately investigated so far, we compared outcomes of patients receiving cabazitaxel with or without daily corticosteroids in a retrospective single-Institution cohort of $\mathrm{mCRPC}$ patients.

Patients and methods: Medical records of deceased patients with documented mCRPC treated with cabazitaxel following prior docetaxel between January, 2011 and January, 2017 were reviewed at the single participating center. Patients who were receiving daily doses of systemic corticosteroids other than low dose daily prednisone or prednisolone (<=10 mg a day) were excluded. The primary end point of this analysis was overall survival (OS). Secondary end-points were exposure to cabazitaxel as well as incidence of grade 3-4 adverse events. Univariable and multivariable Cox proportional hazards regression was used to evaluate prednisone use and other variables as potentially prognostic for overall survival.

Results: Overall, among 91 patients, 57 patients received cabazitaxel concurrently with low dose prednisone and 34 patients did not receive concurrent prednisone. The median overall survival of the population was 9.8 months (interquartile range, 9 to 14). Patients receiving prednisone had an overall survival of 9 months (interquartile range, 8 to 12) vs.14 months (interquartile range, 9.4 to 16.7) for patients not treated with prednisone. Approximately 45\% of patients had a $>30 \%$ PSA decline at 12 weeks. Prednisone use was not significantly prognostic for overall survival or PSA decline $\geq 30 \%$ rates on regression analyses. Importantly, a >30\% PSA decline at 12, but not at 3, 6, 9 weeks, was prognostic for improved survival at multivariate analysis

Conclusions: The data presented here support the hypothesis that omitting daily corticosteroids in cabazitaxel-treated patients has no negative impact on either survival or safety profile. In the large prospective trial CABACARE, cabazitaxel-treated patients will be randomized to receive or not receive daily prednisone. The CABACARE (EudraCT n. 2016-003646-81) study is currently ongoing at University Federico II of Naples and at other multiple participating centers in Italy.
\end{abstract}

Key words: prostate cancer, cabazitaxel, PSA decline. 


\section{Introduction}

Prostate cancer is highly prevalent in men, and an estimated 1,111,700 incident cases were reported in 2012 worldwide [1]. Virtually all patients with recurrent disease after local or loco-regional treatments develop castration-resistant prostate cancer (CRPC), which progresses despite androgen deprivation treatment [2]. The treatment scenario for metastatic CRPC (mCRPC) has been rapidly evolving over the past five years, with novel therapeutic options approved either in the pre- or post-docetaxel setting or both, including one chemotherapy agent (cabazitaxel), two hormonal agents (enzalutamide and abiraterone), one radiopharmaceutical agent (radium-223) and one immunotherapy agent (Sipuleucel-T) [3]. Cabazitaxel is a second-generation taxane that was specifically designed to preserve its effectiveness after onset of resistance to taxanes [4]. In fact, in the TROPIC trial, cabazitaxel was associated with a survival improvement vs. mitoxantrone in patients pretreated with docetaxel [5], although chemotherapy- naïve patients enrolled in the FIRSTANA trial did not show incremental survival benefits with cabazitaxel vs. docetaxel [6]. Of note, limited data suggest that cabazitaxel (vs. hormonal agents) may be particularly efficacious in selected patients, such as those with primary refractoriness to docetaxel [7], brain metastases [8] and those with clinical features of neuroendocrine differentiation [8].

Extensive evidence gathered in expanded access programs (EAPs) has confirmed cabazitaxel efficacy and safety profile [9], with apparently no cumulative toxicity [10]. In both the TROPIC and in the FIRSTANA trials, as well as in the EAPs, cabazitaxel was administered according to its label indication in combination with daily low-dose oral corticosteroids. Nevertheless, whether daily corticosteroids have a role in improving cabazitaxel efficacy or ameliorating its safety profile has not been adequately investigated so far. Corticosteroids may exert both anti-neoplastic and pro-tumorigenic effects, and their potential benefits must be weighed against the known adverse events associated with prolonged use. Interestingly, recent retrospective data indicate that corticosteroids may be associated with beneficial effects in docetaxel-treated patients, but only in those patients who have not previously received corticosteroids [11]. In this retrospective study, we collected data of mCRPC patients treated in a real-world scenario in order to analyze the implications in terms of overall survival, exposure to cabazitaxel and adverse events of omitting daily corticosteroids in patients receiving cabazitaxel.

\section{Patients and methods}

\section{Inclusion criteria}

Medical records of deceased patients with documented mCRPC treated with cabazitaxel following prior docetaxel between January, 2011 and January, 2017 were reviewed at the single participating center. Patients who were receiving daily doses of systemic corticosteroids other than low dose daily prednisone or prednisolone $(<=10 \mathrm{mg}$ a day) were excluded.

\section{Retrieved data}

Demographic data of eligible patients were retrieved along with information regarding known recognized baseline prognostic factors, use of prednisone, previous systemic treatments for mCRPC, as well as early PSA declines $>30 \%$ at 3, 6, 9 and 12 weeks. All patients who received at least one dose of cabazitaxel were included in the dataset.

\section{Data analysis}

The primary end point of this analysis was overall survival (OS). Secondary end-points were exposure to cabazitaxel as well as incidence of grade 3-4 adverse events. Summary statistics were used to describe the patient population and outcomes. In each sub-set, survival was computed from the start of cabazitaxel to the time of death. Univariable Cox proportional hazards regression was used to evaluate variables as potentially prognostic for overall survival. Multivariable modeling was conducted by using the full model method, i.e. including all potential factors in a multivariable model, regardless of whether they were statistically significant or not. The effect of prednisone on survival was then assessed after adjusting for factors included in the multivariable model. The association of prednisone use with exposure to cabazitaxel treatment, measured as the total number of cycles delivered, was tested by the use of Mann-Whitney test. Finally, the association of prednisone use with 30\% PSA declines as well as the onset of grade 3-4 adverse events was tested using Fisher's exact test. All tests were two-sided and a p-value of 0.05 or less was considered statistically significant. No statistical adjustments were made for multiple testing.

\section{Results}

\section{Cohorts' characteristics and outcomes}

Overall, there were 91 patients who received cabazitaxel following prior docetaxel and were not on systemic corticosteroids other than low dose prednisone were included in this analysis (table 1). Fifty-seven patients received cabazitaxel concurrently 
with low dose prednisone and 34 patients did not receive concurrent prednisone. The reason for not receiving corticosteroids was presence of adverse events associated with previous corticosteroid use in 18 patients, contraindication to the use of corticosteroids (diabetes, hypertension) in 9 cases and was unknown in the remaining patients. Approximately half of the population had visceral disease and required opioids to manage pain. The median time from diagnosis to onset of metastatic disease was 15 months (interquartile range 0 to 41 ), while the median time from diagnosis to onset of CRPC was 26 months (interquartile range, 6 to 48,5 ). All patients had received docetaxel, while 23 and 8 patients had received abiraterone and enzalutamide, respectively.

Table 1. Cohort's characteristics

\begin{tabular}{|c|c|c|c|}
\hline & Patients $(\mathrm{N})$ & $\%$ & Total evaluable \\
\hline Gleason Score & & & 81 \\
\hline $6-7$ & 33 & $37.5 \%$ & \\
\hline $8-10$ & 48 & $54.5 \%$ & \\
\hline Opioid use & & & 90 \\
\hline Yes & 45 & $50 \%$ & \\
\hline No & 45 & $50 \%$ & \\
\hline Visceral disease & & & 91 \\
\hline Yes & 44 & $48.46 \%$ & \\
\hline No & 47 & $51.64 \%$ & \\
\hline $\begin{array}{l}\text { Neutrophil / } \\
\text { lymphocyte ratio }\end{array}$ & & & 91 \\
\hline$>=3$ & 34 & $37.36 \%$ & \\
\hline$<3$ & 57 & $62.64 \%$ & \\
\hline $\begin{array}{l}\text { Low dose } \\
\text { prednisone }\end{array}$ & & & 91 \\
\hline Yes & 57 & $62.64 \%$ & \\
\hline \multirow[t]{2}{*}{ No } & 34 & $37.36 \%$ & \\
\hline & Median & $\begin{array}{l}\text { Interquartile } \\
\text { range }\end{array}$ & \\
\hline $\begin{array}{l}\text { Time from } \\
\text { diagnosis to onset } \\
\text { of metastatic } \\
\text { disease (months) }\end{array}$ & 15 & 0 to 41.7 & 91 \\
\hline $\begin{array}{l}\text { Time from } \\
\text { diagnosis to CRPC } \\
\text { (months) }\end{array}$ & 26 & 6 to 48.5 & 91 \\
\hline $\begin{array}{l}\text { Hemoglobin levels } \\
\text { prior to cabazitaxel } \\
\text { (g/dL) }\end{array}$ & 12.7 & 10.4 to 13.6 & 91 \\
\hline Age (years) & 69 & 62 to 72 & 91 \\
\hline
\end{tabular}

\section{Impact of prednisone on Overall survival and PSA declines}

The median overall survival of the population was 9.8 months (interquartile range, 9 to 14). Patients receiving prednisone had a median overall survival of 9 months (interquartile range, 8 to 12) vs.14 months (interquartile range, 9.4 to 16.7) for patients not receiving prednisone. However, prednisone use was not significantly prognostic for overall survival on univariate or multivariate analyses. Six, 8, 27 and 44 patients showed $\geq 30 \%$ PSA declines at $3,6,9$ and 12 weeks, respectively. Differences in terms of $\geq 30 \%$ PSA declines rates were not significant ( $p$ value not shown in table 2) among patients treated with and without prednisone on univariate or multivariate analyses (see table 3). When examining all variables in the entire cohort at univariate analysis, $\geq 30 \%$ PSA declines at 9 and 12 weeks, hemoglobin levels, opioid use were associated with improved overall survival, while at multivariate analysis only younger age (HR per year $=1.03 ; 95 \% \mathrm{CI}=1.00$ to $1.07 ; \mathrm{p}=0.039)$ and $30 \%$ PSA declines at 12 weeks (Hazard Ratio, 0.53; 95\% CI=0.31 to $0.89 ; \mathrm{p}=0.0182$ ) (but not PSA declines at 9 weeks) were associated with improved overall survival (table $3)$.

\section{Impact of prednisone on toxicities}

The median number of cabazitaxel cycles administered was 7 (interquartile range, 3 to 12). Only hemoglobin levels and absence of use of opioids were associated with increased number of cabazitaxel cycles received. Prednisone, Gleason score and PSA declines at 12 weeks were not statistically significant, although trends were observed (table 4). Adverse events reported were those expected on the basis of the existing literature (see table 5). Prednisone was not associated differences in any of the grade 3-4 events reported at univariate or multivariate analysis (data not shown).

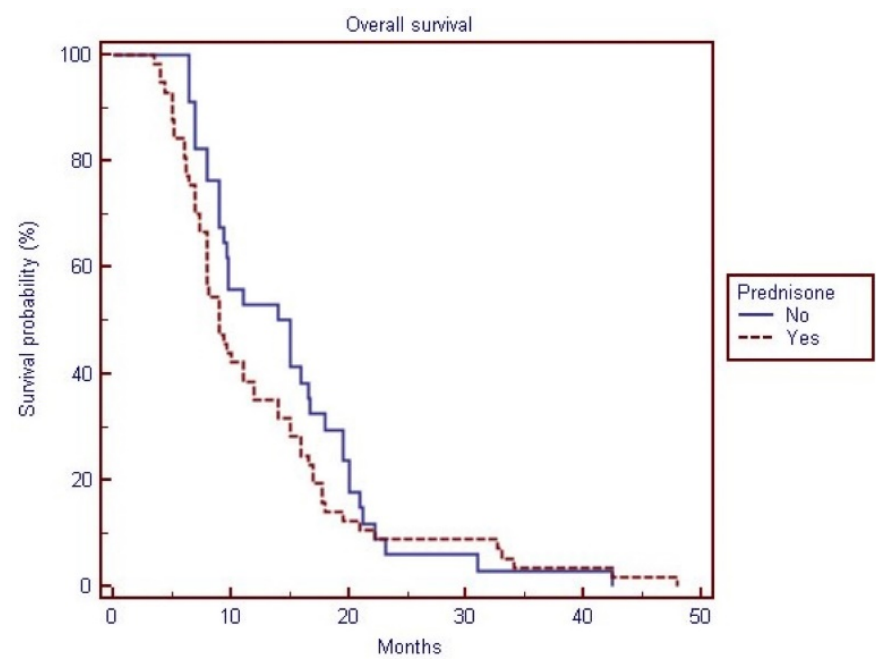

Figure 1. Overall survival of the study population (cabazitaxel with prednisone vs. cabazitaxel alone)

\section{Discussion}

Low-dose corticosteroids may be beneficial in patients with CRPC by suppressing production of weak androgens of adrenal origin via its negative feedback on secretion of adrenocorticotrophic hormone (ACTH). Tannock et al. [12] reported that 
low-dose prednisone treatment (7.5 to $10 \mathrm{mg}$ daily) decreased serum testosterone levels in seven of nine patients who had failed to achieve serum levels below $2.0 \mathrm{nmol} / \mathrm{mL}$, with a decrease in serum levels of androstenedione and dehydroepiandrosterone sulfate in more than $50 \%$ of patients. Furthermore, glucocorticoids may also have direct inhibitory effects on prostate cancer cells through enhanced growth inhibitory TGF- $\$ 1$ signaling and suppression of the transcriptional activities of NF- $\mathrm{kB}$ [13]. Evidence from clinical trials is not conclusive at the present time. In the retrospective review study by Teply et al. [11], 131 men treated with docetaxel plus prednisone and 69 treated with docetaxel alone were stratified according to prior exposure to prednisone (with abiraterone) or hydrocortisone (with ketoconazole) use. Interestingly, patients receiving prednisone had longer progression-free survival compared with the docetaxel-alone cohort (median PFS: 7.8 vs. 6.2 months, HR $0.68 \quad(95 \%$ confidence interval (CI) $0.48-0.97), P=0.03$ ), while no statistically significant advantage was seen among patients pre-treated with abiraterone- or ketoconazole (median PFS: 7.1 vs. 6.3 months, HR 0.96 (95\% CI 0.59-1.57), P=0.87). This finding was not confirmed in the retrospective study by Kongsted et al., who reported no difference in progression-free survival between $234 \mathrm{mCRPC}$ patients treated with docetaxel plus daily corticosteroids vs. 124 patients treated with docetaxel only. Nevertheless, omitting daily prednisone was associated in this study with a higher incidence of peripheral edema $(32 \%$ vs. $15 \%, \mathrm{P}<0.001)$ and febrile neutropenia ( $25 \%$ vs. $10 \%, \mathrm{P}<0.001)$ [14]

Table 2. Cabazitaxel-associated outcomes

\begin{tabular}{|c|c|c|c|c|c|c|c|}
\hline Variable & $\begin{array}{l}\text { All Patients } \\
(\mathrm{N})\end{array}$ & $\%$ & $\begin{array}{l}\text { Patients using } \\
\text { prednisone }(\mathrm{N})\end{array}$ & $\%$ & $\begin{array}{l}\text { Patients not using } \\
\text { prednisone }(\mathrm{N})\end{array}$ & $\%$ & $\begin{array}{l}\text { Total } \\
\text { evaluable }\end{array}$ \\
\hline $30 \%$ PSA decline at 3 weeks & & & & & & & 82 \\
\hline Yes & 6 & $7.2 \%$ & 1 & $3,2 \%$ & 5 & $9,8 \%$ & \\
\hline No & 77 & $91.7 \%$ & 30 & $96,8 \%$ & 46 & $90,2 \%$ & \\
\hline $30 \%$ PSA decline at 6 weeks & & & & & & & 81 \\
\hline Yes & 8 & $9.7 \%$ & 6 & $12.8 \%$ & 2 & 5,9 & \\
\hline No & 74 & $90.2 \%$ & 41 & $87.2 \%$ & 32 & 94,1 & \\
\hline $30 \%$ PSA decline at 9 weeks & & & & & & & 88 \\
\hline Yes & 27 & $30,3 \%$ & 16 & $28.6 \%$ & 13 & $40.6 \%$ & \\
\hline No & 62 & $69,6 \%$ & 40 & $71.4 \%$ & 19 & $59.4 \%$ & \\
\hline $30 \%$ PSA decline at 12 weeks & & & & & & & 91 \\
\hline Yes & 41 & $45,0 \%$ & 23 & 40.4 & 18 & $52.9 \%$ & \\
\hline No & 50 & $54,9 \%$ & 34 & 59.6 & 16 & $47.1 \%$ & \\
\hline Prednisone & & & & & & & 91 \\
\hline No & 34 & $37,3 \%$ & & & & & \\
\hline \multirow[t]{2}{*}{ Yes } & 57 & $62,6 \%$ & & & & & \\
\hline & Median & Interquartile range & Median & Interquartile range & Median & Interquartile range & \\
\hline Cabazitaxel cycles & 7 & 3 to 12 & 5 & 4 to 13 & 10 & 3 to 11,25 & 91 \\
\hline Overall Survival & 9.8 & 9 to 14 & 9 & 8 to 12 & 14 & 9.4 to 16.7 & 91 \\
\hline
\end{tabular}

Table 3. Univariate and multivariate analysis of prognostic factors

\begin{tabular}{|c|c|c|c|c|c|}
\hline \multicolumn{2}{|l|}{ Factor } & \multirow{2}{*}{$\begin{array}{l}\mathbf{N} \\
91\end{array}$} & \multicolumn{2}{|c|}{ Hazard Ratio $(95 \% \mathrm{CI})$} & \multirow{2}{*}{$\begin{array}{l}\text { p-value } \\
0.0816\end{array}$} \\
\hline Age at Cabazitaxel treatment initiation & / year & & 1.01( & 03) & \\
\hline Hemoglobin treatment baseline & / unit & 91 & 0.87 & $.94)$ & 0.0010 \\
\hline $30 \%$ PSA decline at 3 weeks & Yes vs. No & 82 & 1.45( & 36) & 0.3866 \\
\hline $30 \%$ PSA decline at 6 weeks & Yes vs. No & 81 & 1.28( & $68)$ & 0.5017 \\
\hline $30 \%$ PSA decline at 9 weeks & Yes vs. No & 91 & 0.59( & 93) & 0.0236 \\
\hline $30 \%$ PSA decline at 12 weeks & Yes vs. No & 91 & 0.43( & 67) & 0.0003 \\
\hline Neutrophils/Lymphocyte Ratio & $>=3$ vs. $<3$ & 91 & 1.45( & 25) & 0.0924 \\
\hline Gleason Score & $\geq 8$ vs $\leq 7$ & 81 & 0.72( & 12) & 0.1531 \\
\hline Visceral Disease & Yes vs No & 91 & 1.50( & 29) & 0.0588 \\
\hline Opiates & Yes vs No & 89 & 1.97( & 09) & 0.0032 \\
\hline Prednisone & Yes vs. No & 91 & 1.27( & 96) & 0.2603 \\
\hline \multicolumn{6}{|l|}{ Multivariable Model } \\
\hline Age at Cabazitaxel initiation & / year & 78 & 1.03 & 1.00 to 1.07 & 0.0398 \\
\hline Hemoglobin at treatment baseline & / unit & & 0.97 & 0.87 to 1.07 & 0.5699 \\
\hline$>30 \%$ PSA decline at 12 weeks & Yes vs. No & & 0.53 & 0.31 to 0.89 & 0.0182 \\
\hline Neutrophils/Lymphocyte Ratio & $>=3$ vs. $<3$ & & 1.48 & 0.85 to 2.57 & 0.1644 \\
\hline Gleason Score & $\geq 8$ vs $\leq 7$ & & 0.59 & 0.33 to 1.03 & 0.068 \\
\hline Opiates treatment baseline & Yes vs No & & 1.66 & 0.98 to 2.79 & 0.0555 \\
\hline Prednisone & Yes vs No & & 1.46 & 0.89 to 2.41 & 0.1279 \\
\hline Visceral Disease at treatment baseline & Yes vs No & & 1.30 & 0.76 to 2.22 & 0.3211 \\
\hline
\end{tabular}


Table 4. Median number of cycles and baseline variables

\begin{tabular}{|c|c|c|c|}
\hline & & $\begin{array}{l}\text { Median number } \\
\text { of cycles }\end{array}$ & P value \\
\hline Age at Cabazitaxel initiation & $>=69$ vs. $<69$ & 5 vs. 8 & $\mathrm{P}=0.3357$ \\
\hline $\begin{array}{l}\text { Hemoglobin at treatment } \\
\text { baseline }\end{array}$ & $>=12$ vs. $<12$ & 10 vs. 4 & $\mathrm{P}=0.0008$ \\
\hline$>30 \%$ PSA decline at 12 weeks & Yes vs. No & 10 vs. 5 & $\mathrm{P}=0.0570$ \\
\hline $\begin{array}{l}\text { Neutrophils/Lymphocyte } \\
\text { Ratio }\end{array}$ & $>=3$ vs. $<3$ & 5 vs. 10 & $P=0.1525$ \\
\hline Gleason Score & $>=8$ vs. $<7$ & $8 \mathrm{Vs} .4$ & $P=0.0845$ \\
\hline Opiates treatment baseline & Yes vs. No & 4 vs.9 & $P=0.0442$ \\
\hline Visceral Disease & Yes vs. No & 5 vs. 9 & $P=0.3255$ \\
\hline Prednisone & Yes vs. No & 5 vs. 10 & $P=0.0569$ \\
\hline
\end{tabular}

Table 5. Overall incidence of severe adverse events.

\begin{tabular}{ll}
\hline Adverse events & $\begin{array}{l}\text { Number of patients } \\
(\%)(\mathrm{n}=91)\end{array}$ \\
\hline Grade 3-4 neutropenia & $27(29.67 \%)$ \\
Grade 3-4 anemia & $4(4.39 \%)$ \\
Febrile neutropenia & $3(3.29 \%)$ \\
Grade 3-4 Leukopenia & $7(7.69 \%)$ \\
\hline
\end{tabular}

When administered alone, prednisone was associated with a palliative effect in $12 \%$ of patients in a randomized phase III trial comparing mitoxantrone plus prednisone vs. prednisone [15]. In randomized studies of abiraterone, the use of prednisone alone demonstrated measurable clinical benefits including PSA declines $(10 \%$ after docetaxel, $24 \%$ in docetaxel-naïve men) and Response Evaluation Criteria in Solid Tumours (RECIST) responses (3\% and $16 \%$, respectively) [16, 17]. Nevertheless, no advantage in OS was associated with the use of single-agent daily prednisone [18].

The question whether prednisone should be added to systemic anti-neoplastic treatment in men with CRPC is of particular importance in patients receiving cabazitaxel. In fact, a patient may have been exposed to daily prednisone for 1-2 years in combination with abiraterone given in the first-line setting and then for approximately 6 months in combination with docetaxel, before receiving cabazitaxel. Adverse events, such as hypertension and hyperglycemia associated with prolonged use (>1year) of corticosteroids may have an impact on the quality of life [19]. Furthermore, prolonged exposure to corticosteroids may select AR variants that make tumors sensitive to glucocorticoids even at low concentrations. Point mutations of the AR, occurring in the ligand-binding domain in most cases, are rare in therapy- naive patients but are reported in 15\% to $45 \%$ of castration-resistant disease and can increase AR affinity for a wide range of steroids. Over 100 mutations have been reported and many be associated with a functional advantage in maintaining $A R$ signaling [20]. Finally, corticosteroids negatively affect lipid metabolism and increase fat deposition [21], which are both related to increased risk of prostate cancer progression and death [22]. In a docetaxel-pretreated population such as that enrolled in the AFFIRM trial, prednisone had a detrimental effect in terms of OS [23]. In this study, omitting corticosteroids was not associated with a negative impact on survival. On the contrary, there was a trend at multivariate analysis towards shorter overall survival in patients who were treated with corticosteroids $(\mathrm{HR}=1.27 ; 95 \% \mathrm{CI}=0.83$ to 1.96 ; $\mathrm{p}=$ 0.26 ), and a decreased exposure to cabazitaxel was associated with daily corticosteroid use (median number of cycles, 5 vs. 10). Interestingly, we also found that patients experiencing a 30\% PSA decline as early as 12 weeks had improved OS at multivariate analysis, similarly to docetaxel [24]. Of note, 30\% PSA declines at 3, 6 and 9 weeks were not prognostic at multivariate analysis, possibly due to the effect of PSA kinetics of early cytolysis induced by cabazitaxel [25]. Finally, we noted that patients with a higher Gleason score appeared to receive a greater number of cabazitaxel cycles (8 vs. 4). Such a difference was borderline significant, but it is consistent with previously published data suggesting an association of higher Gleason score with longer progression-free survival [26].

The study presented here presents the limitations typical of a retrospective study, including the lack of sample size calculation and data incompleteness. Furthermore, patients receiving $>10$ $\mathrm{mg}$ prednisone or an equivalent dose of other corticosteroids were excluded from this analysis, so the findings reported may not be applied to patients with a more advanced disease treated with systemic corticosteroids. Nevertheless, the data presented here support the hypothesis that omitting daily corticosteroids in cabazitaxel-treated patients may have no negative impact on either survival or cabazitaxel safety profile. In this regard, a large, prospective phase IIb trial CABACARE, which is currently ongoing at University Federico II of Naples and at other multiple participating Italian centers, will randomize approximately 220 patients to cabazitaxel with or without daily prednisone.

\section{Conclusions}

In conclusion, this study did not identify any detrimental effect associated with omission of daily corticosteroids in patients receiving cabazitaxel, in terms of either safety or efficacy. In a population of patients treated with cabazitaxel for prostate cancer, who are likely to have received daily corticosteroids for $>1$ year, omission of daily corticosteroids may be beneficial. In this regard, the data presented here can 
serve as background to support the conduction of adequately designed prospective trials assessing safety and efficacy of cabazitaxel with or without daily corticosteroids.

\section{Acknowledgments}

This work was partially supported by the LILT (Lega Italiana per la Lotta ai Tumori) Sez. Napoli.

\section{Competing Interests}

Carlo Buonerba \& Giuseppe Di Lorenzo: Research Support to Institution from Astellas, Sanofi and Quercegen Pharmaceuticals, personal fees from for Sanofi (unrelated to this work)

Guru Sonpavde: Grants from BoehringerIngelheim, grants from Bayer, grants from Onyx-Amgen, personal fees from Pfizer, personal fees from Genentech, personal fees from Novartis, personal fees from Argos, grants and personal fees from Merck, personal fees from Sanofi, personal fees from Agensys, personal fees from Clinical Care Options, personal fees from Astrazeneca, personal fees from Uptodate, personal fees from Biotheranostics, personal fees from Exelixis, personal fees from Bristol-Myers-Squibb, personal fees from Janssen, personal fees from Amgen, personal fees from Eisai, personal fees from NCCN (National Comprehensive Cancer Network), outside the submitted work.

\section{References}

1. Torre LA, Bray F, Siegel RL et al. Global cancer statistics, 2012. CA Cancer J Clin 2015; 65: 87-108.

2. Buonerba C, Di Lorenzo G, Sonpavde G. Contemporary molecular tests for prognosis and treatment guidance for castration-resistant prostate cancer. Expert Rev Mol Diagn 2016; 16: 1113-1120.

3. Kapoor A, Wu C, Shayegan B, Rybak AP. Contemporary agents in the management of metastatic castration-resistant prostate cancer. Can Urol Assoc J 2016; 10: E414-E423.

4. Vrignaud P, Semiond D, Benning V et al. Preclinical profile of cabazitaxel. Drug Des Devel Ther 2014; 8: 1851-1867.

5. de Bono JS, Oudard S, Ozguroglu M et al. Prednisone plus cabazitaxel or mitoxantrone for metastatic castration-resistant prostate cancer progressing after docetaxel treatment: a randomised open-label trial. Lancet 2010; 376: $1147-1154$

6. A. Oliver Sartor SO, Lisa Sengelov. Cabazitaxel vs docetaxel in chemotherapy-naive $(\mathrm{CN})$ patients with metastatic castration-resistant prostate cancer (mCRPC): A three-arm phase III study (FIRSTANA). J Clin Oncol 2016; 34 (suppl): abstr5006.

7. Di Lorenzo G, Bracarda S, Buonerba C et al. Poor survival in prostate cancer patients with primary refractoriness to docetaxel. Eur Urol 2014; 65: 505-507.

8. Di Lorenzo G, Buonerba C, de Placido S. Rapidly progressive disease in a castration-resistant prostate cancer patient after cabazitaxel discontinuation. Anticancer Drugs 2015; 26: 236-239.

9. Bracarda S, Gernone A, Gasparro D et al. Real-world cabazitaxel safety: the Italian early-access program in metastatic castration-resistant prostate cancer. Future Oncol 2014; 10: 975-983.

10. Di Lorenzo G, Bracarda S, Gasparro D et al. Lack of Cumulative Toxicity Associated With Cabazitaxel Use in Prostate Cancer. Medicine (Baltimore) 2016; 95: e2299.

11. Teply BA, Luber B, Denmeade SR, Antonarakis ES. The influence of prednisone on the efficacy of docetaxel in men with metastatic castration-resistant prostate cancer. Prostate Cancer Prostatic Dis 2016; 19: 72-78.

12. Tannock I, Gospodarowicz M, Meakin W et al. Treatment of metastatic prostatic cancer with low-dose prednisone: evaluation of pain and quality of life as pragmatic indices of response. J Clin Oncol 1989; 7: 590-597.
13. Nishimura $\mathrm{K}$, Nonomura $\mathrm{N}$, Satoh $\mathrm{E}$ et al. Potential mechanism for the effects of dexamethasone on growth of androgen-independent prostate cancer. J Natl Cancer Inst 2001; 93: 1739-1746.

14. Kongsted P, Svane IM, Lindberg $\mathrm{H}$ et al. Low-dose prednisolone in first-line docetaxel for patients with metastatic castration-resistant prostate cancer: is there a clinical benefit? Urol Oncol 2015; 33: 494 e415-420.

15. Tannock IF, Osoba D, Stockler MR et al. Chemotherapy with mitoxantrone plus prednisone or prednisone alone for symptomatic hormone-resistant prostate cancer: a Canadian randomized trial with palliative end points. J Clin Oncol 1996; 14: 1756-1764.

16. Ryan CJ, Smith MR, de Bono JS et al. Abiraterone in metastatic prostate cancer without previous chemotherapy. N Engl J Med 2013; 368: 138-148.

17. de Bono JS, Logothetis CJ, Molina A et al. Abiraterone and increased survival in metastatic prostate cancer. N Engl J Med 2011; 364: 1995-2005.

18. Sonpavde G, Pond GR, Templeton AJ et al. Impact of single-agent daily prednisone on outcomes in men with metastatic castration-resistant prostate cancer. Prostate Cancer Prostatic Dis 2017; 20: 67-71.

19. Hougardy DM, Peterson GM, Bleasel MD, Randall CT. Is enough attention being given to the adverse effects of corticosteroid therapy? J Clin Pharm Ther 2000; 25: 227-234.

20. Zhao XY, Malloy PJ, Krishnan AV et al. Glucocorticoids can promote androgen-independent growth of prostate cancer cells through a mutated androgen receptor. Nat Med 2000; 6: 703-706.

21. Asensio C, Muzzin P, Rohner-Jeanrenaud F. Role of glucocorticoids in the physiopathology of excessive fat deposition and insulin resistance. Int J Obes Relat Metab Disord 2004; 28 Suppl 4: S45-52.

22. Ferro $M$, Terracciano $D$, Buonerba $C$ et al. The emerging role of obesity, diet and lipid metabolism in prostate cancer. Future Oncol 2017; 13: 285-293.

23. Scher H I FK, Saad F et al. Impact of on-study corticosteroid use on efficacy and safety in the Phase III AFFIRM study of enzalutamide (ENZA), an androgen receptor inhibitor. Presented at: American Society Clinical Oncology (ASCO), Genitourinary Cancers Symposium. Orlando, FL, USA, 14-16 February (2013)

24. Armstrong AJ, Tannock IF, de Wit $\mathrm{R}$ et al. The development of risk groups in men with metastatic castration-resistant prostate cancer based on risk factors for PSA decline and survival. Eur J Cancer 2010; 46: 517-525.

25. Angelergues A, Maillet D, Flechon A et al. Prostate-specific antigen flare induced by cabazitaxel-based chemotherapy in patients with metastatic castration-resistant prostate cancer. Eur J Cancer 2014; 50: 1602-1609.

26. Buonerba C, Pond GR, Sonpavde G et al. Potential value of Gleason score in predicting the benefit of cabazitaxel in metastatic castration-resistant prostate cancer. Future Oncol 2013; 9: 889-897. 